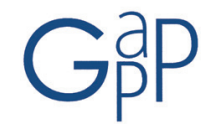

GESTIÓN Y ANÁLISIS DE POLÍTICAS PÚBLICAS, Nueva Época, nº 13 enero-junio 2015 ISSN: 1989-8991

DOI: http://dx.doi.org/10.24965/gapp.voi13.10237

\title{
El Índice de Cobertura de Servicios (ICS). Un instrumento para la evaluación de los Servicios Públicos'
}

\author{
Nieves Lagares Diez \\ Universidad de Santiago de Compostela \\ mnieves.lagares@usc.es \\ María Pereira López \\ Universidad de Santiago de Compostela \\ mariapereira.lopez@gmail.com \\ Erika Jaráiz Gulías \\ Universidad de Santiago de Compostela \\ erika.jaraiz@usc.es
}

\section{Resumen}

El amplio crecimiento experimentado por los servicios públicos en España en los últimos treinta y ocho años, paralelo a la estructuración de las administraciones públicas, al proceso de descentralización de competencias y al desarrollo del Estado del Bienestar; ha puesto de manifiesto la importancia que su cobertura tiene no sólo para las administraciones públicas, como gestoras de los mismos, sino para los ciudadanos, como copropietarios últimos de dichos servicios.

Bajo el soporte teórico que proporcionan los presupuestos del paradigma del Nuevo Servicio Público, se pone en valor la noción de ciudadanía, así como los derechos inherentes a la misma, colocando al ciudadano en el centro del debate público. Para conseguir estos objetivos, las administraciones deben poner en marcha mecanismos que les permitan conocer las expectativas y el grado de satisfacción de los ciudadanos con los servicios que prestan. Para ello y teniendo en cuenta el bagaje teórico-empírico en este terreno, se ha diseñado una propuesta teórica, el Índice de Cobertura de Servicios (ICS), que permite analizar de forma comparativa, a través del establecimiento de umbrales de cobertura, el nivel de prestación de los servicios públicos en el ámbito municipal.

Palabras clave

Índice de Cobertura de Servicios, umbrales de cobertura, indicadores, satisfacción ciudadana, modelos de ecuaciones estructurales.

\section{Index of Services Coverage (ISC). A tool for assessment of Public Service}

\section{Abstract}

The comprehensive growth experienced by the public services in Spain in the last thirty-eight years, parallel to the structuring of public administrations, to the process of decentralization of powers and to the development of the welfare State; It has become apparent the importance of its coverage not only for public administrations, as managers, but for citizens as co-owners of such services.

Under support theoretical that provide the budgets of the paradigm of the New Public Service, put in value the notion of citizenship, as well as the rights inherent thereto, by placing the citizen at the centre of public debate. To achieve these objectives, administrations must put in place, mechanisms that allow them to know the expectations and satisfaction of citizens with the services provided. Do this and taking into account the theoretical and empirical background in this area, has been designed a theoretical proposal, the rate of coverage of services (ICS), that allows to analyze in a comparative way, through the establishment of thresholds for coverage, the level of coverage of the public services at the municipal level.

Key words

Index of Services Coverage, coverage thresholds, indicators, citizen satisfaction, structural equation modeling.

1 Nuestro más sincero agradecimiento a la Diputación de A Coruña, la realización de esta investigación no habría sido posible sin su apoyo a través de diferentes proyectos. Agradecemos también a los gestores y técnicos de la misma, así como a los técnicos de los ayuntamientos que han estado implicados desde el primer momento en este proyecto. 


\section{INTRODUCCIÓN}

En los últimos años, la necesidad de control y evaluación periódica de las acciones llevadas a cabo por los diferentes departamentos públicos, así como el uso de los recursos públicos destinados a dichos fines, ha cobrado especial relevancia. El concepto de evaluación de la gestión pública, importado de las filosofías del New Public Management primero y posteriormente del New Public Service, trata de dar respuesta a las demandas de mayor control, transparencia y responsabilidad de los asuntos públicos por parte de la ciudadanía. Una ciudadanía cada vez más activa y que reclama mayor protagonismo en la gestión de los servicios públicos de los cuáles es y se siente agente activo.

En este contexto, se vuelve imprescindible dotar a los responsables de la gestión pública de instrumentos para el análisis de la prestación de los servicios públicos, que les permitan realizar evaluaciones rigurosas y objetivas, antes, durante y después de la puesta en marcha de políticas, proyectos y/o acciones; calibrando de esta forma el impacto que las medidas puestas en marcha y los recursos públicos destinados a las mismas, tienen sobre los usuarios directos e indirectos de dichos servicios. Surge así la necesidad de construir nociones y conceptos que nos permitan comprender este impacto, definirlo, objetivarlo y medirlo; entre ellos, el concepto de satisfacción ciudadana con los servicios públicos, el cual se ha convertido en elemento central sobre el que pivotará la evaluación de la prestación de los servicios públicos.

La creación e implantación de este tipo de instrumentos o herramientas de medición puede suponer una importante estructura de oportunidad no sólo para el seguimiento de las políticas públicas puestas en marcha, sino también para la retroalimentación continua de la relación gestor-usuario; que permitirá identificar los outputs y outcomes positivos y/o negativos que el sistema genera, para potenciar los primeros y eliminar, sino reducir en la medida de lo posible, el impacto de los segundos. La necesidad de estos mecanismos de mejora y optimización en la prestación de los servicios públicos si bien es importante en todos los niveles administrativos, se vuelve vital en el caso del ámbito municipal.

Es en este contexto donde surge la construcción de un instrumento de medición, inusual y novedoso, de la prestación o cobertura de los servicios públicos, el Índice de Cobertura de Servicios (ICS). Un índice que se perfila como una herramienta de gestión estratégica para los responsables políticos y los gestores públicos de las entidades locales y que permite, al mismo tiempo, mejorar los mecanismos que los ciudadanos tienen a su disposición para incrementar el conocimiento sobre los servicios.

\section{LA SATISFACCIÓN CIUDADANA}

Satisfacción y calidad percibida son dos conceptos que han estado siempre íntimamente relacionados, hasta el punto de que en ocasiones se han llegado a usar como sinónimos (Jaráiz Gulías, E. , 2011). Con un desarrollo teórico paralelo, ambos constructos han sido difíciles de definir y diferenciar; desde quienes ven en la satisfacción un componente más de la calidad percibida, pasando por aquellos que consideran que la satisfacción se ve condicionada por aquella, hasta llegar a quienes conjugan ambas posturas; entendiendo la satisfacción como una consecuencia de la calidad percibida del servicio, cuya evaluación - la de la calidad percibida - estaría a su vez condicionada por la satisfacción experimentada en los diferentes encuentros de servicio previos (Parasuraman, A.; Zeithmal, V. y Berry, L., 1994a) (Oliver R. , 1994) (Martínez-Tur, V., Peiró Silla, J.M. y Ramos, J., 2001). A pesar de ser similares y compartir algunos elementos, no podemos afirmar por ello, que sean términos polivalentes; girando el debate en torno a la relación existente entre las expectativas y percepciones del usuario acerca del servicio, y no sobre un criterio objetivo predeterminado sobre lo que es o debería ser el servicio.

El concepto de satisfacción es extremadamente complejo, por lo que su estudio y análisis se ha abordado desde diferentes entornos y disciplinas, las cuáles han intentado arrojar algo de luz sobre los elementos que la componen, sobre su funcionamiento y sobre las consecuencias que tiene para la gestión estratégica de los servicios. Prueba de la amplia cantidad de definiciones existentes, así como de la evolución que el propio concepto ha sufrido; es el hecho de que podamos encontrarnos en la literatura especializada, con hasta treinta y siete definiciones distintas de satisfacción desde el año 1969, década - la de los años sesenta - en la que algunos autores sitúan el inicio del estudio de este concepto (Giese, J. L. y Cote, J. A., 2000) (Gil Saura, I. et al., 2004), de la mano de los trabajos de Howard y Sheth (1969), mientras que otros lo retrasan hasta la década de los setenta (Evrard, 1993), con los trabajos de Day o Hunt (1977) 
Teniendo en cuenta el amplio volumen de definiciones existentes, podemos estructurar la evolución que ha sufrido el debate en torno a la satisfacción y sus componentes, en el cual se ha ido enriqueciendo y matizando el concepto, en base a la discusión que se ha vertebrado en torno a dos enfoques principales: por un lado la concepción de la satisfacción como resultado o outcome y por otro, la concepción de la satisfacción como proceso o performance. El primero de estos dos enfoques, el enfoque basado en el resultado o outcome, fuertemente influenciado por la perspectiva economicista, entiende el estudio de la satisfacción como el resultado de la transacción de bienes o servicios. En esta línea se encuentran los primeros trabajos de Day (1977), Hunt (1977) u Oliver (1980); afirmando que el consumidor obtiene su satisfacción cuando al comparar las percepciones con las expectativas que albergaba sobre la prestación del servicio, el resultado obtenido de dicha comparación supera sus expectativas (desconfirmación positiva), obteniéndose como resultado un consumidor satisfecho; en caso contrario, cuando el resultado percibido es menor que sus expectativas (desconfirmación negativa), el consumidor se encontrará insatisfecho (Miller, 1977) (Oliver, R. , 1980) (Tse, D. y Wilton, P., 1988) (Bitner, 1990). Con posterioridad estos autores evolucionarían el sentido de sus definiciones de satisfacción influenciados por la psicología, lo que les llevaría a introducir un componente afectivo (Westbrook, 1980) (Westbrook, R.,A. y Reilly, D., 1983) (Oliver R. , 1981). Este último llevaría la noción de afectividad hasta el punto de definir la satisfacción como la respuesta a la saciedad del cliente (Oliver R. L., 1997).

Por su parte, el enfoque fundamentado en el proceso o performance, se fundamenta en el llamado paradigma de la desconfirmación de expectativas, en el cual el usuario realiza una evaluación entre sus percepciones sobre los resultados del servicio que ha recibido y un estándar de comparación, generalmente las expectativas que previamente se había creado sobre la prestación del servicio (Oliver, R. , 1981). O en palabras de Zeithmal y Bitner (2002), la evaluación que realiza el cliente respecto de un producto o servicio, en términos de si ese producto o servicio respondió a sus necesidades y expectativas. En otros trabajos, se han propuesto estándares de comparación diferentes a las expectativas, es el caso de la experiencia pasada (Cadotte, E.; Woodruff, R. y Jenkins, R., 1987), basada en anteriores encuentros de servicio; los deseos inherentes al usuario (Spreng, R.,A. y MacKoy, R.,D., 1996) o la comparación social de costes-beneficios (Oliver, R.L. y Swan, J.,E., 1989). Más recientemente, autores tradicionalmente cercanos al paradigma del resultado o performance, han profundizado en el concepto y han ido derivando hacia propuestas integradoras donde los factores cognitivos y afectivos se entremezclan (Westbrook, R.,A. y Reilly, D., 1983) (Oliver, R.,L. y Swan, J.E., 1989) (Oliver, R. , 1993) (Spreng, R.,A. y MacKoy, R.,D., 1996) (Richard, M.,D. y Allaway, A.,W., 1993); entendiendo la satisfacción como una respuesta afectiva o emocional, producto de las emociones experimentadas en los sucesivos encuentros o experiencias de servicio.

A modo de conclusión, podemos decir que cada vez es mayor el consenso respecto de que los dos conceptos - calidad y satisfacción - son, en esencia o por naturaleza (Oliver, R. , 1993b) (Rust, R. T. y Oliver, R. L., 1994) (Anderson, E. W.; Fornell, C. y Lehmann, D. R., 1994), distintos en términos de las causas subyacentes, así como de sus resultados (Parasuraman, A.; Zeithmal, V. y Berry, L., 1994a); si bien tienen ciertos aspectos en común, la satisfacción por lo general se observa como un concepto más amplio, mientras que la evaluación de la calidad en el servicio se centra específicamente en las dimensiones del mismo. Desde este punto de vista, la percepción de la calidad con el servicio es un componente de la satisfacción del usuario (la calidad como predecesora de la satisfacción) se encuentran los trabajos de Cronin y Taylor (1992); aunque la calidad con el servicio es una evaluación dirigida que refleja las percepciones del usuario sobre ciertas dimensiones específicas del servicio: confiabilidad, responsabilidad, seguridad, empatía y tangibles. Por su parte, la satisfacción es más inclusiva, influyen sobre ella las percepciones de la calidad del servicio, la calidad del producto y el precio, así como factores situacionales y personales (Zeithmal, V. y Bitner, M.J., 2002). En la línea de considerar la satisfacción como antecedente de la calidad destacan los trabajos de Bolton y Drew (1991) o Bitner (1990); finalmente algunos autores proponen una visión integradora y circular de la concepción de ambos términos, Oliver (1994), Parasuraman et al. (1994a), Rust y Oliver (1994) o Teas (1993).

Si bien entre ambas tradiciones, norteamericana y europea, existen semejanzas como la concepción de la calidad de servicio de la que parten o la consideración de dos grandes tipos de dimensiones (tangible e intangible), también existen importantes diferencias. En cualquier caso, en este trabajo la satisfacción se entiende como un concepto fundamental a la hora de analizar y evaluar la prestación o cobertura de los servicios públicos (Jaráiz Gulías, E. , 2009), intentando minimizar en la medida de lo posible, la carga afectiva y emotiva que conlleva; contrarrestándola para ello, con la inclusión de elementos de carácter objetivo. Así pues, en la metodología que aquí se presenta, se entremezclan los datos procedentes de indicadores objetivos públicos y no publicados de cobertura 
de servicios públicos, con datos procedentes de encuestas de satisfacción con dichos servicios. Trataremos en profundidad estas cuestiones metodológicas más adelante.

\section{FORMAS DE MEDICIÓN DE LA SATISFACCIÓN CIUDADANA}

\section{Las escalas clásicas de satisfacción}

Dada la importancia que, como hemos puesto de relieve, tiene el estudio y medición de la calidad y la satisfacción del usuario para las organizaciones, sean éstas privadas o públicas y para la calidad de vida en general; es comprensible que a partir de finales de los años ochenta comenzaran a proliferar algunas iniciativas en diferentes países - tanto en el sector público como en el sector privado - , que tenían por finalidad, medir los niveles de calidad y de satisfacción de los usuarios con los servicios.

La mayor parte de estas iniciativas se basaron en la elaboración de estudios demoscópicos, los cuales permitían medir cuestiones relativas a aquellas características del servicio que determinaban el grado de satisfacción/ insatisfacción del usuario del mismo; así como la calidad subjetiva de los servicios que ofrecían las organizaciones e incluso la relación entre las expectativas que los usuarios habían puesto en la prestación de un servicio y los resultados finales que recibían. Si bien estos estudios y concretamente las encuestas que se elaboraban para llevarlos a cabo, gozaron de una gran aceptación tanto en el ámbito privado como en el ámbito público con la aplicación a casos concretos, no es menos cierto que también han recibido algunas críticas. Entre estas críticas o problemas que presentaban estos instrumentos de medición se han destacado, la dificultad para elaborar una lista exhaustiva de las características de un servicio, única y válida para todos los usuarios; que los cuestionarios de este tipo hayan resultado ser en la práctica, excesivamente largos e incluso que los resultados obtenidos a través de la aplicación de los mismos, pequen de cierta subjetividad, revelándose como juicios de valor de los diferentes usuarios sobre sus propios gustos o preferencias (Oliver R. , 1997)2.

Algunas de estas iniciativas se materializaron en la construcción de modelos de encuestas, es el caso de la encuestas SERVQUAL (Parasuraman, A.; Zeithmal, V. y Berry, L., 1988), la encuesta SERVPERF (Cronin, J.J. y Taylor, S., 1992) o la encuesta E-S-QUAL (Parasuraman, A.; Zeithmal, V. y Malhotra, A., 2005); así como en índices nacionales de satisfacción de los usuarios, a través de los cuales se medía y se daba seguimiento a la calidad y grado de satisfacción del cliente o usuario de servicios en el nivel macroeconómico.

Ampliamente criticada, la escala SERVQUAL generó un amplio debate en la literatura sobre calidad y satisfacción del servicio, dando lugar a numerosas réplicas, bien usando esta escala o sus correspondientes revisiones y/o redefiniciones o bien proponiendo medidas o escalas alternativas como es el caso de Cronin y Taylor (1992) (1994) a principios de los años noventa, con la presentación del modelo alternativo, SERVPERF, o las críticas de Carman (1990) o Babakus y Boller (1992). Esta encuesta sufrió varios procesos de redefinición por parte de los autores, dando lugar al denominado modelo de los gaps (Parasuraman, A., Zeithmal, V. y Berry, L., 1991) (1994a) (1994b); quienes ante las limitaciones metodológicas identificadas, procedieron a una adaptación del instrumento a las nuevas dimensiones de la calidad del servicio que habían adquirido relevancia (Parasuraman, A.; Zeithmal, V. y Malhotra, A., 2005). Esta nueva escala, denominada E-S-QUAL, refleja las dimensiones de eficiencia, disponibilidad del sistema, cumplimiento y privacidad y está indicada para la medición de la calidad en ambientes en línea.

Paralelamente al desarrollo de este debate, comienzan a proliferar a partir de los años ochenta y noventa, los Índices de Satisfacción del Consumidor o Customer Satisfaction Index (CSI). Construidos en base a potentes herramientas de análisis estadístico y basados en teorías o aproximaciones teóricas, permitían conocer el comportamiento del consumidor, la identificación de los condicionantes de su satisfacción con los servicios, así como las consecuencias que se podían derivar de una potencial insatisfacción del usuario del servicio o el nivel de calidad del servicio (Fornell, 1992).

Una de las primeras iniciativas en este sentido, fue la llevada a cabo por Suecia, con la creación del Barómetro Sueco de la Satisfacción del Cliente (Swedish Customer Satisfaction Barometer, SCSB) que se introdujo en el año 1989, un nuevo sistema de medida de las 32 mejores compañías de este país (Fornell, 1992). En 1991, se publicaba

2 Estos problemas se han tenido muy en cuenta en nuestra investigación a la hora de elaborar el cuestionario que hemos aplicado en los estudios demoscópicos, tanto en lo relativo a su formato, con la determinación de las preguntas, la extensión de la entrevista o el orden y redacción de las respuestas; como en cuestiones de fondo, eliminando la subjetividad implícita en este tipo de conceptos, no sólo en la redacción de las preguntas evitando en todo momento preguntar por características del servicio, sino por datos objetivos de cobertura del mismo, o seleccionando los formatos de respuesta, optando mayoritariamente por el empleo de una única escala numérica (o-10) para la codificación. Esta decisión metodológica ha permitido no sólo obtener unas respuestas muchas más sistemáticas, sino que también ha facilitado la explotación y análisis de los resultados. 
en Alemania, el Barómetro de Satisfacción del Cliente (Deutsche Kundenbarometer, DK) y tres años más tarde, veía la luz el Índice Estadounidense de Satisfacción del Cliente (American Customer Satisfaction Index, ACSI) (Fornell, C.; Johnson, M. D.; Anderson, E. W.; Cha, J. y Everitt Bryant, B., 1996) (CFI, 2008). En 1996 se presentaría el Índice Noruego de Satisfacción (Norwegian Customer Satisfaction Index, NCSB) (Andreassen, T. W. y Lervik, L., 1999) y en el año 2000, el Índice Europeo de Satisfacción del Consumidor (European Customer Satisfaction Index, ECSI) (Eklof, 2000). Todos estos índices supusieron un importante avance, permitiendo comprender las motivaciones de la satisfacción de los consumidores y sirviendo como mecanismos para la mejora de la calidad de los servicios en diferentes sectores. Además, han servido de importante base teórica y empírica para la elaboración de metodologías posteriores, como la que nos ocupa.

\section{Catálogos de indicadores e índices de satisfacción}

En el terreno del análisis de los servicios públicos, así como de la satisfacción ciudadana con dichos servicios, son muchas y variadas, las experiencias empíricas concretas que se han llevado a cabo.

Por un lado, nos encontramos con las experiencias llevadas a cabo por diferentes organizaciones internacionales y nacionales que han puesto en marcha diversos catálogos o baterías de indicadores de gestión³. La mayoría de estas organizaciones han llevado a cabo la elaboración de indicadores de gestión para las entidades públicas, particularmente en el ámbito local; incidiendo en el problema de la tipología de indicadores que deben utilizarse, así como en los objetivos que los mismos debieran cumplir. Los principales problemas que resaltan organizaciones como el GASB o la Audit Commision guardan relación con la medición de prestaciones públicas y con el establecimiento de objetivos, así como con la necesidad de resaltar la importancia que debe tener el desarrollo de indicadores de impacto o outcomes (Navarro Galera, A.; Ortiz Rodríguez, D. y López Hernández, A.M., 2006)para el análisis de los servicios públicos.

En lo concerniente a las experiencias basadas en la realización de estudios demoscópicos en España, las más importantes en relación con el análisis de las actitudes y percepciones de los ciudadanos, así como con el grado de satisfacción ciudadana con los servicios públicos, han sido realizadas bajo la batuta del Observatorio de la Calidad de los Servicios Públicos de la Agencia Estatal de Evaluación de las Políticas Públicas y la Calidad de los Servicios (AEVAL), en colaboración con el Centro de Investigaciones Sociológicas (CIS) (AEVAL, 2014). Desde su creación, el CIS, bien a través de baterías de preguntas dentro de encuestas generales como es el caso de los barómetros o bien como estudios de carácter específico, ha llevado a cabo trabajos de gran calidad sobre estas cuestiones (Van Ryzin, G. G. y DelPino, E., 2009); marcando una tendencia dentro del ámbito académico en España.

Experiencias similares a las descritas, pueden ser encontradas a nivel internacional, tal es el caso de los Eurobarómetros, elaborados por el departamento de Análisis de la Opinión Pública de la Comisión Europea desde 1974 o la encuesta Urban Audit sobre satisfacción con los servicios urbanos. Otras experiencias destacadas, la encuesta de carácter nacional en la que se evalúa la satisfacción ciudadana con la prestación de servicios procedentes del sector público, Citizen First, que elabora en Canadá el Institute for Citizen-Centered Service (ICCS); la National Citizen Survey o Encuesta Nacional Ciudadana elaborada en EE.UU. por el National Research Center (NRC) o Centro Nacional de Investigación y la Asociación Internacional de Gestión Provincial y Municipal (ICMA) o las encuestas que se han llevado a cabo en ciudades del Reino Unido sobre sectores de servicios concretos al amparo del principio del Best Value.

Finalmente, destacan los modelos mixtos que buscan la elaboración de índices sintéticos a partir de dos fuentes de información: los catálogos de indicadores y la aplicación de cuestionarios específicos. El European Primer on Customer Satisfaction (ECPSM), propuesto por la UE en el año 2008 es un ejemplo de este tipo de metodología. Este índice aspira a convertirse en un instrumento que aúne metodologías entre los estados miembros de la Unión Europea (UE) para el estudio de la satisfacción ciudadana con los servicios públicos ${ }^{4}$. Ya en España, podemos encontrar experiencias, que han permitido la construcción de índices agregados de medición de la calidad o satisfacción con los servicios públicos a nivel autonómico. Entre ellos se encuentran: el Índice de Percepción de la Calidad de los Servicios (IPCS) elaborado por la Junta de Extremadura y el Observatorio de la Calidad de los Servicios desde 2005; el Índice de Percepción de Calidad del Servicio elaborado por el Observatorio de la Calidad de Madrid, que

3 Entre estas experiencias pueden destacarse los trabajos del GASB (Governmental Accounting Standard Board), la ANAO (Australian National Audit), la Audit Commision, la AECA (Asociación Española de Contabilidad y Administración de Empresas); o los trabajos desarrollados en un nivel administrativo inferior por parte de la Diputación de Barcelona o la Diputación de A Coruña.

4 EI ECPSM ha sido el resultado de la colaboración entre la European Public Administration Network (EUPAN) y el European Institute of Public Administration (EIPA) a petición de la Presidencia portuguesa de la UE. 
ha elaborado un estudio periódico desde 1997 o el Barómetro de Satisfacción de la Ciudadanía dentro de la Estrategia de Modernización de los Servicios Públicos de la Junta de Andalucía (2006-2010). Mención especial merece la "Propuesta de Indicadores básicos de Gestión de Servicios Públicos Locales", catálogo de indicadores elaborado por varias instituciones catalanas ${ }^{5}$, donde se recogen indicadores estructurados en siete grandes áreas temáticas ${ }^{6}$ y para cuya elaboración se crearon varios grupos de trabajo en los que participaron expertos en el ámbito administrativo, así como miembros de algunas de las entidades locales participantes (Batet i Lamaña, M. et al., 2001) (Batet Lamaña, M. y Mora Puigví, A., 2001). En esta misma línea destaca el proyecto SINIGAL (Sistema Normalizado de Indicadores de Gestión para la Administración Local)7 cuyo objetivo es la normalización de baterías de indicadores para algunos servicios municipales y cuya experiencia se llevó a cabo en algunos ayuntamientos andaluces (López, A. M.; Navarro Galera, A. y Ortiz, D., 2001) (López Hernández, A. y Ortiz Rodríguez, D., 2004) (Navarro Galera, A.; Ortiz Rodríguez, D. y López Hernández, A.M., 2006). También de interés es el proyecto elaborado para el estudio de la valoración de los factores determinantes de la calidad del servicio público local percibido por los ciudadanos y sus repercusiones sobre su satisfacción, cuya aplicación en la práctica se circunscribió a los pequeños municipios de Castilla y León; y en el que se utilizaron tanto datos obtenidos a través de internet o de boletines de la Junta de Castilla y León, como datos de carácter privado y datos procedentes de la elaboración de una encuesta específica sobre calidad y satisfacción (Gutiérrez Rodríguez, P. y García-Inés, M., 2008). Por último, señalar el Barómetro de Gestión Municipal, elaborado por el Equipo de Investigaciones Políticas de la Universidad de Santiago de Compostela, con el patronato de la Diputación de A Coruña.

De los estudios llevados a cabo por estas organizaciones se derivan importantes conclusiones en relación a las limitaciones o problemas que se pueden encontrar en la implantación de sistemas normalizados de indicadores de gestión en las administraciones públicas; si bien, a pesar de todos ellos y teniendo en cuenta que todas estas experiencias presentan importantes diferencias metodológicas, en cuanto a la recolección y análisis de datos; nadie pone en duda la importancia que tiene en la actualidad el análisis y evaluación de la gestión de las políticas, acciones y servicios públicos, independientemente del paradigma teórico que los inspire o de la forma metodológica elegida.

\section{UN ÍNDICE INUSUAL: EL ÍNDICE DE COBERTURA DE SERVICIOS}

\section{Construcción del Î́ndice de Cobertura de Servicios}

Partiendo de la base existente en la literatura teórica y empírica al respecto nace esta investigación, con la finalidad de crear una metodología mixta de análisis de los servicios públicos municipales que ha desembocado en la construcción del denominado, Índice de Cobertura de Servicios, un índice sintético resultado de una compleja formulación matemática y de un profundo análisis estadístico previo, que ha permitido su desarrollo y testeo a nivel municipal, concretamente en los ayuntamientos de la provincia de A Coruña (Pereira López, 2013).

Contextualizado dentro de la óptica teórica del New Public Service y lo que esto conlleva en términos de transparencia, rendición de cuentas y responsabilidad; el ICS se presenta como un instrumento de análisis de cobertura, fundamentado sobre un balance equilibrado entre los elementos estructurales que definen el servicio y los elementos perceptivos que los ciudadanos expresan sobre la prestación de cada servicio. Por ello, el Índice de Cobertura de Servicios, no es en sentido estricto un índice de satisfacción tal y como lo interpreta la literatura clásica, sino un índice de cobertura en el que la satisfacción se tiene en cuenta como un elemento importante, pero como uno más del mismo.

Los elementos estructurales del servicio vienen representados por los datos procedentes de los indicadores objetivos públicos y no publicados recogidos mediante trabajo de campo en cada uno de los ayuntamientos que se desean analizar. Estos indicadores conforman un cuadro de mando integral de análisis de los servicios públicos municipales, que permite determinar un análisis general sobre la situación de cada uno de los ayuntamientos, sirviendo de punto de partida para el análisis. La incorporación al esquema de estos indicadores dota de realismo a la medición de la satisfacción ciudadana, al tiempo que permite identificar más fácilmente desviaciones respecto al nivel medio de satisfacción, así como posibles déficits en la cobertura y prestación de los servicios.

5 Diputación de Barcelona, Sindicatura de Comptes, Fundación Carles Pi i Sunyer de Estudios Autonómicos y Locales y Colegio de Economía de Cataluña.

6 Indicadores de perfil de ciudad, indicadores de servicios sociales, indicadores de servicios personales, indicadores de atención al ciudadano, indicadores de limpieza viaria, indicadores de recogida de residuos sólidos urbanos e indicadores de alumbrado.

7 Este proyecto es fruto de la colaboración entre la Universidad de Granada, la Cámara de Cuentas de Andalucía y la Federación Andaluza de Municipios y Provincias. 
Por su parte, los elementos perceptivos que componen el índice vienen definidos por las variables incluidas en estudios demoscópicos sobre satisfacción. Desde el primer momento se tuvo claro que dentro del ámbito de "lo público", se hacía imprescindible para su análisis, proceder a la inclusión de la medición de la satisfacción ciudadana con los servicios públicos como un elemento fundamental del instrumento de medida (Jaráiz Gulías, E., Lagares Diez, N. y Pereira López, M., 2013). Esta visión permite tener en cuenta las expectativas y preferencias que los ciudadanos tienen sobre los servicios públicos en general y sobre los elementos que los componen en particular.

El hecho de tener en cuenta ambos elementos o fuentes de información - datos objetivos y encuestas de satisfacción (figura 1) -, evita caer en una mera descripción de la situación de los servicios a nivel municipal, así como en una eventual desconexión de las percepciones y expectativas, y por tanto, de los niveles de satisfacción expresados por los ciudadanos. En consecuencia, la conjugación planteada es altamente enriquecedora de cara a la construcción del indicador y de cara al análisis del nivel de satisfacción ciudadana, del grado de cobertura de las áreas y servicios prestados y del desempeño de las entidades locales.

El índice que proponemos en esta investigación constituye pues, un mecanismo de evaluación que permite identificar los elementos de los servicios públicos que tienen un mayor o menor impacto sobre la satisfacción que expresan sobre cada uno de ellos y sobre la satisfacción general en su conjunto; así como conocer la situación real de cobertura de los servicios en cada uno de los ayuntamientos en términos comparados y promover la mejora de los elementos que se desvelan como críticos dentro del esquema, con la finalidad de alcanzar mayores niveles de satisfacción, equidad y eficiencia en la prestación y cobertura de los servicios públicos en cada ayuntamiento.

\section{FIGURA 1. ELEMENTOS Y RELACIONES EN LA CONSTRUCCIÓN DEL ÍNDICE DE COBERTURA DE SERVICIOS PÚBLICOS}

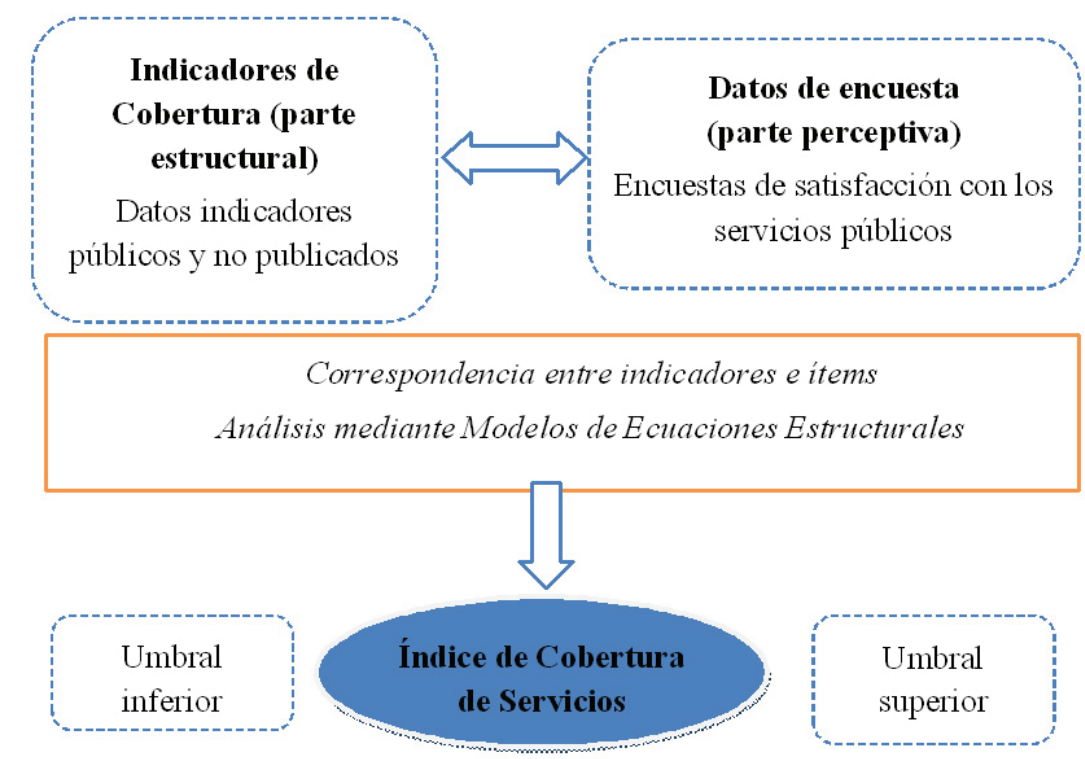

Fuente: elaboración propia.

Si tuviéramos que definir el ICS diríamos que es un índice que permite expresar el grado de cobertura de los servicios públicos que prestan los municipios, poniendo en relación dimensiones de carácter objetivo, elemento estructural, con la priorización que los ciudadanos hacen de la incidencia de los componentes internos que definen a estos servicios, con los niveles de satisfacción con dichos servicios expresados por ellos mismos, elemento perceptivo.

Fundamentando el índice en las dos bases de información comentadas, los indicadores de diagnóstico municipal y la evaluación de la percepción ciudadana sobre los servicios públicos municipales, se construye una herramienta metodológica que permite: una sistematización de la información de especial interés municipal, una identificación de los elementos claves con mayor incidencia en la satisfacción ciudadana, una identificación de debilidades en los servicios para cada uno de los municipios y una visión comparativa entre ayuntamientos del mismo tamaño poblacional. 
Para hacer posible esta visión comparativa, la construcción y aplicación (Pereira López, 2013) del ICS se planificó teniendo en cuenta una clasificación en base al tamaño poblacional de los municipios que iban a ser objeto de estudio. Esta decisión se tomó porque no se buscaba obtener estimaciones generales de los resultados, sino estimaciones específicas de la situación por la que atravesaban los servicios públicos en cada uno de los ayuntamientos y de forma comparativa, en relación a los municipios de su mismo tamaño o franja poblacional. Es necesario precisar, que incluso entre ayuntamientos de la misma franja poblacional, existen diferencias importantes en lo que a recursos, medios y criterios de gestión y prestación de servicios se refiere, por lo que los niveles de satisfacción de los ciudadanos, obviamente, no tienen por qué mostrar similitudes; por ello, es imprescindible disponer de un diagnóstico preciso y particular de los distintos servicios en cada una de las poblaciones. Delimitadas las fuentes de información y obtenidos los datos a través de cada una de ellas, cabe preguntarse por cuál ha sido la forma de poner en relación aspectos en principio tan dispares.

En la mayor parte de los casos la relación que se ha planteado entre los indicadores objetivos y los ítems de la encuesta, resulta evidente, puesto que miden exactamente lo mismo que se pretende evaluar. En aquellos casos donde la correspondencia no es tan clara, la relación se ha establecido por proximidad temática con el elemento interno objeto de análisis. Además, en algunas ocasiones la relación que se establece es múltiple, es decir, un ítem puede estar relacionado con uno o más indicadores objetivos. La correspondencia establecida entre ítems de la encuesta e indicadores objetivos ha sido la misma para todos los ayuntamientos, independientemente de su tamaño poblacional; puesto que la finalidad última es proceder a realizar un análisis comparado de cobertura de servicios.

En la elaboración de este tipo de metodologías es conveniente seguir ciertos pasos: 1) desarrollo de un marco teórico, 2) selección de los indicadores originales, 3) análisis estadístico multivariante, 4) imputación de los valores y casos perdidos, 5) normalización de los datos, 6) distribución de pesos y agregación, 7) relación entre los indicadores con otras variables, 8) análisis de los datos reales y 9) presentación de los resultados (Nardo, M.et al., 2005) (Nardo, M.et al., 2005). A lo largo de la investigación hemos seguido en gran medida este esquema, siendo reflejo de ello, la presentación del marco teórico relativo a los servicios públicos y a la satisfacción; la elección de los indicadores objetivos públicos y no publicados; la realización del análisis estadístico multivariante, a través de ecuaciones estructurales para cada uno de los servicios y para la satisfacción en su conjunto, previo al cual se han imputado los valores perdidos de nuestras bases de datos; y finalmente, el establecimiento, a través del cuadro de mando integral creado con los indicadores objetivos, las relaciones de éstos con los ítems de la encuesta. En este sentido, dos de los pasos fundamentales previos a la presentación de la fórmula que permite el cálculo del ICS, fueron la normalización de los datos y la distribución de pesos.

En cuanto al primero de ellos, la normalización de los datos procedentes de los indicadores objetivos, se vuelve imprescindible para avanzar en la construcción del índice de cobertura, dado que resulta habitual que dichos datos estén medidos en diferentes escalas e incluso en diferentes unidades de medida. Para ello se decidió optar por la estandarización de los datos como técnica para la normalización de los mismos ${ }^{8}$; de esta forma se evita la inclusión de posibles distorsiones en el momento de agregación de los indicadores objetivos en cada uno de los componentes internos que caracterizan a los servicios.

Normalizados los datos, se procede al cálculo y distribución de pesos para cada uno de los elementos; distribución que se establece automáticamente en virtud de la correspondencia previamente establecida entre indicadores objetivos e ítems de la encuesta de satisfacción. Los pesos se obtienen a través de la aplicación en cada una de las bases de datos (para cada franja poblacional) de la técnica estadística de análisis factorial confirmatorio mediante componentes principales. Se decidió optar por aplicar esta técnica por ser una de las más empleadas para la determinación de pesos y por permitir reducir la dimensionalidad de los datos, a partir de la identificación de factores latentes o indicadores compuestos mediante agregación jerárquica de componentes ${ }^{9}$. Posteriormente, los pesos de los elementos internos de cada servicio se obtienen a través de un cálculo realizado en base a los

8 Una vez transformadas las puntuaciones de todos los indicadores objetivos en puntuaciones estandarizadas o puntuaciones $Z$, se puede describir con precisión la posición relativa de un individuo dentro de la distribución; así como también conocer la probabilidad asociada a cualquier valor x de una variable normalmente distribuida, a partir de la probabilidad asociada a su correspondiente puntuación z-score.

9 Estos factores creados en base a las correlaciones existentes entre las variables objetivas, dependen de un grupo de coeficientes o cargas factoriales que miden la correlación entre el indicador original y el factor latente. A los factores latentes se les ha aplicado una rotación ortogonal que consigue minimizar el número de indicadores originales con cargas factoriales elevadas en más de un factor; simplificándose considerablemente su interpretación y permitiéndose identificar qué indicadores originales saturan en cada uno de los factores latentes extraídos. 
valores obtenidos mediante el análisis factorial previo para dichos elementos, dentro de cada factor.

Llegados a este punto, se operacionaliza, de acuerdo con la correspondencia establecida con anterioridad entre los ítems de la encuesta y los indicadores, de modo que se pueda atribuir a los indicadores objetivos normalizados la ponderación que les corresponde en la construcción de la satisfacción ciudadana, en función de los datos que se obtienen de la realización del mencionado análisis factorial. Se evidencia de esta forma, la importancia que en la construcción del ICS tiene cada una de las bases o fuentes de información obtenidas, los indicadores objetivos y los resultados de las encuestas de satisfacción.

La expresión matemática final, correspondiente al valor del Índice de Cobertura de Servicio (ICS $)$ es la siguiente:

$$
\mathrm{ICS}_{\mathrm{k}}=\left(\sum \mathrm{TP}_{\mathrm{ji}}\right) / 2
$$

donde ICS serán los k índices de cobertura de servicio calculados y $\sum \mathrm{TP}_{\mathrm{ji}}$ representa el sumatorio de todos los totales parciales asociados a dicho servicio ${ }^{10}$. El cociente permite determinar las desviaciones positivas o negativas del propio índice. Finalmente, se reconduce el valor final del índice al intervalo $(0,1)$, hecho que se buscó especialmente para facilitar la lectura de los resultados obtenidos.

El cálculo de estos valores permitirá un posterior análisis que conducirá, ineludiblemente, a establecer comparaciones a través del benchmarking entre ayuntamientos pertenecientes a una misma franja poblacional. Análisis en el que además, se pueden identificar claramente cuáles son los componentes internos de los servicios con mayor impacto en la satisfacción o insatisfacción ciudadana, al tiempo que se pone el foco sobre las desviaciones que presentan los indicadores referentes a la cobertura del servicio respecto de la cobertura media de los ayuntamientos con cargas poblacionales similares. El Índice de Cobertura de Servicios resultante de las operaciones anteriormente descritas, es producto de una serie de cálculos que incorporan diferentes tipologías de indicadores, simples y complejos, y cuyo valor oscila siempre en el intervalo 0-1, donde o significaría el menor nivel de cobertura y 1 el mayor nivel de cobertura del servicio.

En la arquitectura de este índice agregado intervienen una parte fija y común a todos los ayuntamientos de una misma franja poblacional, que es la que procede del análisis de la encuesta, o lo que es lo mismo, de la medición de la satisfacción ciudadana (elemento perceptivo); y una parte variable, procedente de los indicadores reales asociados a cada ayuntamiento y que definen el estado de cobertura de los servicios públicos prestados en cada uno de ellos y que han sido incluidos en el estudio (elemento estructural).

Una vez operacionalizados los datos, es posible estimar los umbrales inferior y superior, que sirven de referencia para establecer el rango de cobertura de servicios entre el que deben de moverse los ayuntamientos. De este modo, un ayuntamiento cuyo índice para un área o servicio determinado estuviese por debajo del umbral inferior establecido para los ayuntamientos de su tamaño poblacional, debería mejorar la prestación de dicha área o servicio, ya que la satisfacción de los ciudadanos con el mismo se situaría en un nivel crítico. Por el contrario, aquellos ayuntamientos cuyo índice supera el umbral superior para un área o servicio determinado, muestran un grado de satisfacción ciudadana con el servicio mucho más elevado del que tienen los demás ayuntamientos de su mismo tamaño de hábitat. La delimitación de umbrales de cobertura de un servicio permite hacer referencia a los niveles mínimos a partir de los cuáles los servicios, programas o actividades públicas ofrecidas por las entidades locales son percibidas y recibidas de forma satisfactoria por los ciudadanos; la identificación de las disfunciones o déficits en la prestación de los servicios permitirá a los gestores públicos poner especial atención sobre los mismos. La construcción de los umbrales mínimos y máximos se realiza para cada servicio o área en cada una de las franjas poblacionales, y no se trata de una simple delimitación de límites superiores o inferiores de la distribución del ICS en dicha franja poblacional, sino de un cálculo matemático a partir de desviaciones a la media.

El establecimiento de umbrales para el índice de cobertura tiene un valor fundamental para la construcción metodológica, pues a través de ellos se identifican aquellos servicios que presentan una mayor o menor incidencia en la satisfacción de los usuarios con los mismos, permitiendo de esta forma mejorar la gestión de los recursos destinados a cada uno de los servicios, pues se evidencia la necesidad o no de redistribución de los recursos públicos destinados a cada uno de los servicios, hacia aquellas áreas de gestión municipal cuyo análisis haya expresado o puesto de manifiesto la existencia de deficiencias.

El resultado final tras la aplicación de la metodología descrita a lo largo de estas líneas, lleva a la creación de una ágil, simple y fiable herramienta estadística de trabajo, que estudia y evalúa la satisfacción ciudadana para ges-

10 Definimos el total parcial correspondiente a cada componente interno del servicio como el sumatorio de todas las puntuaciones estandarizadas asociadas a él, ponderadas por el nivel de satisfacción ciudadana con dicho componente. 
tionar la cobertura de los servicios en el ámbito municipal, suponiendo, por esto último, una importante novedad en lo que a la tradición de las investigaciones empíricas en este terreno se refiere.

\section{Funcionamiento del Índice de Cobertura de Servicios}

La metodología detallada en líneas precedentes ha tenido su aplicación práctica en los resultados recogidos en el Barómetro de Gestión Municipal o puesto en marcha por la Diputación de A Coruña y el Equipo de Investigaciones Políticas de la Universidad de Santiago de Compostela a lo largo del año 2013. En este proyecto se ha Ilevado a cabo el análisis de dieciocho áreas o servicios de gestión municipal en los 94 municipios que componen la provincia de A Coruña"1.

Las áreas o servicios analizados en este estudio fueron definidos por el Equipo en base a la literatura e investigaciones empíricas de referencia y a los resultados obtenidos de la realización de grupos de discusión en los que participaron técnicos de gestión municipal de la provincia de A Coruña. En la figura 2 se presentan los servicios analizados, entre los cuales, como identificará el lector, se encuentran algunos de competencia no municipal. La razón por la cual se decidió incluir este tipo de áreas, independientemente del reparto de competencias que sobre las mismas exista entre los diferentes niveles administrativos, viene motivada principalmente por el hecho de ser, la satisfacción o insatisfacción del ciudadano, un valor esencial y parte fundamental en la construcción del índice.

\section{FIGURA 2. ÁREAS O SERVICIOS PÚBLICOS ANALIZADOS EN EL BARÓMETRO DE GESTIÓN MUNICIPAL DE LA PROVINCIA DE A CORUÑA}

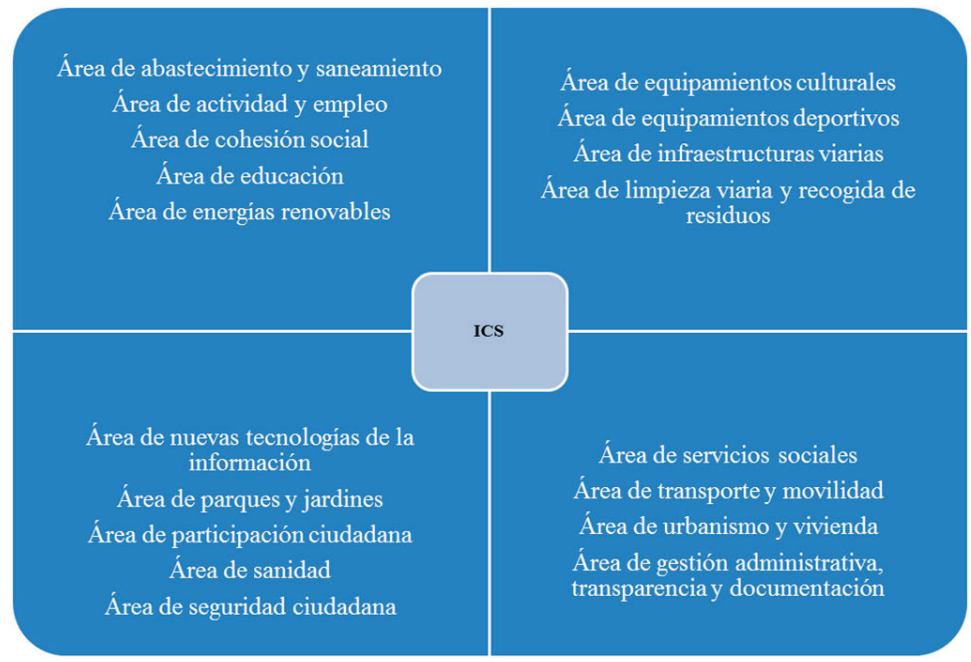

Fuente: elaboración propia.

TABLA 1. REPARTO POBLACIONAL DE LOS MUNICIPIOS OBJETO DE ESTUDIO

\begin{tabular}{|l|l|l|}
\hline Hábitat & Tamaño poblacional & $N^{\circ}$ de municipios \\
\hline Hábitat 1 & Menos de 2.000 habitantes & 12 \\
\hline Hábitat 2 & De 2.001 a 5.000 habitantes & 29 \\
\hline Hábitat 3 & De 5.001 a 20.000 habitantes & 42 \\
\hline Hábitat 4 & De 20.001 a 50.000 habitantes & 8 \\
\hline Hábitat 5 & Más de 50.000 habitantes & 3 \\
\hline
\end{tabular}

Fuente: elaboración propia.

Como se mencionó en líneas precedentes, el análisis de estas áreas o servicios se llevó a cabo en el total de municipios que componen la provincia, agrupados en base al tamaño poblacional, entendiendo que diferentes tamaños poblacionales implican diferentes características sociales, económicas..., y por tanto, diferentes niveles de prestación y recursos para los servicios públicos municipales. Así pues, se dividieron los municipios en cinco grupos o tamaños de hábitat tal y como se puede observar en la tabla 1.

11 En la actualidad el proyecto sigue vigente y se ha llevando a cabo el análisis del Barómetro de Gestión Municipal 1, cuyos resultados serán presentados próximamente. 
Los resultados obtenidos de este proyecto, presentados públicamente el pasado mes de junio, han arrojado interesantes conclusiones sobre la prestación que de los diferentes servicios llevan a cabo los ayuntamientos, así como la prestación de cada uno de ellos en base a los niveles medios de prestación de los municipios con idénticas características. Por ello, podemos afirmar que el ICS permite obtener dos visiones sobre la cobertura que de los servicios públicos municipales llevan a cabo las diferentes corporaciones:

- Una visión individual, que permite determinar los valores del índice en cada servicio para un ayuntamiento concreto, identificando los elementos internos a cada servicio que tienen una mayor o menor incidencia, positiva o negativa, sobre dicho valor; así como su posición en relación a los municipios de su mismo tamaño poblacional.

- Una visión agregada, que facilita una lectura transversal del comportamiento de los servicios analizados en los diferentes tamaños poblacionales. Identificando servicios o áreas que presentan coberturas más o menos homogéneas o heterogéneas en función del tamaño de hábitat.

En la tabla 2, se puede observar un ejemplo de los valores del ICS obtenidos para un municipio de la provincia de A Coruña, ejemplo de la primera visión, la visión individual. Observando con detenimiento las tres primeras filas de resultados de la tabla, nos podemos encontrar con unos valores que definen de forma muy clara y concisa las tres posibilidades que se pueden derivar de la aplicación del índice.

En la primera línea, área de abastecimiento y saneamiento, nos encontramos con un valor del índice que se encuentra por debajo del umbral inferior definido (ICS =0,367; $[0,410,0,596]$ ), por lo que se podría afirmar que el nivel de cobertura del servicio mencionado que presta este ayuntamiento no es adecuado y por tanto resulta insatisfactorio. En cambio, en la segunda línea, se puede observar como el valor del índice en el caso del área de actividad y empleo (ICS = 0,667; [0,446, 0,681], se sitúa entre los umbrales definidos, por lo que se podría decir que el servicio prestado en este caso presenta una cobertura adecuada. Finalmente, en la tercera línea, se presenta el valor del índice para el área de cohesión social (ICS = 0,740; [0,432, 0,692]), un valor notablemente superior al umbral superior, por lo que el servicio prestado en este municipio presenta una cobertura muy notable y satisfactoria.

TABLA 2. VALORES DEL ICS PARA LAS DIECIOCHO ÁREAS O SERVICIOS ANALIZADOS EN UN MUNICIPIO TIPO

\begin{tabular}{|llll|}
\hline Área o Servicio & Valor ICS & Umbral Inferior & Umbral Superior \\
\hline A1.- Área de Abastecimiento y Saneamiento & 0,367 & 0,410 & 0,596 \\
\hline A2.- Área de Actividad y Empleo & 0,667 & 0,446 & 0,681 \\
\hline A3.- Área de Cohesión Social & 0,740 & 0,432 & 0,692 \\
\hline A4.- Área de Energías Renovables & 0,538 & 0,388 & 0,582 \\
\hline A5.- Área de Equipamientos Culturales & 0,696 & 0,400 & 0,665 \\
\hline A6.- Área de Equipamientos Deportivos & 0,544 & 0,411 & 0,575 \\
\hline A7.- Área de Educación & 0,663 & 0,337 & 0,618 \\
\hline A8.- Área de Infraestructuras Viarias & 0,597 & 0,441 & 0,622 \\
\hline A9.- Área de Limpieza Viaria e Recogida de Residuos & 0,660 & 0,380 & 0,636 \\
\hline A10.- Área de Nuevas Tecnologías de la Información & 0,657 & 0,454 & 0,632 \\
\hline A11.- Área de Parques y Jardines & 0,554 & 0,418 & 0,636 \\
\hline A12.- Área de Participación Ciudadana & 0,622 & 0,473 & 0,658 \\
\hline A13.- Área de Sanidad & 0,682 & 0,513 & 0,693 \\
\hline A14.- Área de Seguridad Ciudadana & 0,859 & 0,468 & 0,815 \\
\hline A15.- Área de Servicios Sociales & 0,447 & 0,402 & 0,695 \\
\hline A16.- Área de Transporte y Movilidad & 0,470 & 0,432 & 0,640 \\
\hline A17.- Área de Urbanismo y Vivienda & 0,590 & 0,462 & 0,608 \\
\hline A18.- Área de Gestión Administrativa, Transparencia y Documentación & 0,670 & 0,422 & 0,650 \\
\hline
\end{tabular}

Fuente: elaboración propia.

Si bien y como se mencionó anteriormente, el Índice de Cobertura de Servicios también nos facilita una lectura transversal de la cobertura de cada servicio, que permite anticipar la existencia de diferentes tipos de cobertura, en función de los niveles alcanzados para cada servicio en los diferentes tamaños de hábitat. Por un lado áreas que presentan una cobertura muy heterogénea de los servicios en base al tamaño poblacional, como puede ser el caso 
del área de limpieza viaria y recogida de residuos; y por otro lado, áreas con unos niveles de cobertura homogéneos, caso del área de equipamientos culturales ${ }^{12}$.

Estas dos perspectivas nos permiten plantear una visión conjunta de la cobertura que de los servicios públicos Ilevan a cabo los ayuntamientos, de una forma comparada y teniendo en cuenta dos elementos complementarios, por un lado un elemento de carácter estructural, variable en función de cada ayuntamiento (indicadores del servicio) y por otro un elemento de carácter perceptivo (datos de satisfacción ciudadana), común a todos los ayuntamientos de una misma franja poblacional.

\section{Evolución del Índice de Cobertura de Servicios}

Algunas de las revisiones teórico-empíricas realizadas en los últimos años en relación al análisis de las percepciones de los ciudadanos y la expresión de las mismas a través de niveles de satisfacción, han llevado a recomendar abiertamente, iniciar una línea de análisis y evaluación de la satisfacción no de forma aislada, sino de forma continuada en el tiempo; producto de la naturaleza misma del objeto de estudio, las percepciones y expectativas de los ciudadanos en relación a un servicio. Percepciones y expectativas, que como bien han expuesto autores como Parasuraman, Zeithmal y Berry (1991) no son estáticas sino dinámicas y altamente variables a medio y largo plazo.

Como se ha explicado, la metodología presentada para el análisis de los servicios públicos municipales tiene como uno de sus objetivos principales, la vocación de continuidad en el tiempo. De ello se deduce la necesidad de continuar testando el modelo en nuevos entornos espacio-temporales, que permitan perfilar y mejorar en la medida de lo posible, el Índice de Cobertura de Servicios planteado; así como continuar recopilando información y realizando un seguimiento en aquellos entornos en los que ya haya sido puesto en práctica, que permitan la construcción de sólidas bases de datos de diagnóstico municipal.

Es la aplicación continuada, la que permitirá hacer de esta metodología una herramienta consistente, válida y ágil para el análisis y seguimiento de la cobertura de los servicios públicos por parte de los administradores y gestores municipales. Además, su adecuada y correcta interpretación permitirá llevar a cabo un análisis eficaz y responsable de los recursos públicos de cada uno de los ayuntamientos, reconsiderando la redistribución que de los mismos se ha llevado a cabo hasta el momento, para optimizar en la medida de lo posible los recursos existentes y el nivel de satisfacción ciudadana con la prestación de los mismos, priorizando aquellos servicios que hayan presentado elementos críticos en el análisis.

Una de las líneas futuras a las que debiera conducir esta investigación es al establecimiento de un cuadro de mando de indicadores no sólo de inputs sino también de outputs, que permitan evaluar los niveles de consecución de los objetivos establecidos. Esta información facilitará llevar a cabo no sólo un análisis de resultados sino también un análisis en términos de eficiencia, y por lo tanto, en términos de rendimiento de las inversiones que los organismos municipales y de otro rango administrativo destinan a los primeros para el desarrollo y prestación de determinados servicios o actividades públicas.

Así pues, partiendo del escenario que hemos dibujado sobre los niveles de cobertura de los servicios y teniendo en mente aspectos presupuestarios y de contabilidad de los servicios y del rendimiento obtenido, la evolución natural de los índices de cobertura sería el diseño y desarrollo de índices de rendimiento que evalúen la eficiencia de los recursos invertidos. El objetivo de futuro pasa por testar si la eficiencia en las inversiones realizadas en los diferentes servicios se traduce en un incremento de la eficacia, o lo que es lo mismo, en un incremento del índice de cobertura, dando un paso más en la gestión estratégica de los recursos y fondos públicos.

Manteniendo la línea avanzada por el Índice de Cobertura de Servicios, el diseño y desarrollo de índices de objetivos lleva a impulsar la puesta en práctica de nuevos instrumentos metodológicos destinados a los gestores públicos con el fin de introducir mejoras en la optimización de los recursos y la planificación estratégica con dos objetivos claros: por un lado responder a las expectativas y exigencias de la ciudadanía y por otro, evaluar el rendimiento de las inversiones destinadas a la mejora de los servicios ofrecidos en los ayuntamientos. Determinando de esta forma, si las inversiones realizadas inciden sobre los elementos internos del servicio que el ICS identifica como críticos ${ }^{13}$ y si dichas actuaciones tienen una incidencia positiva en los niveles de satisfacción ciudadana con dichos servicios. Es en este punto, a la hora de evaluar las inversiones realizadas, donde cobra verdadera importancia la identificación que de los elementos internos de los servicios que tienen un mayor o menor impacto sobre la

12 Por cuestión de espacio no se incluyen en este artículo los gráficos correspondientes al análisis transversal por área o servicio.

13 Se emplea la palabra "crítico" exenta de negatividad, pues define a aquellos elementos o componentes internos de un servicio prestado por un determinado municipio y que inciden más directamente en la satisfacción ciudadana con dicho servicio, sea dicha incidencia en sentido positivo o negativo. 
satisfacción con los mismos ha realizado el ICS. Por tanto, el estudio del rendimiento de las inversiones realizadas por los organismos permitirá llevar a cabo una planificación estratégica centrada en una redistribución más eficaz y eficiente de los recursos, permitiendo equilibrar la balanza de gestión de dichas instituciones (figura 2).

FIGURA 3. RELACIONES ÍNDICES DE COBERTURA E ÍNDICES DE RENDIMIENTO

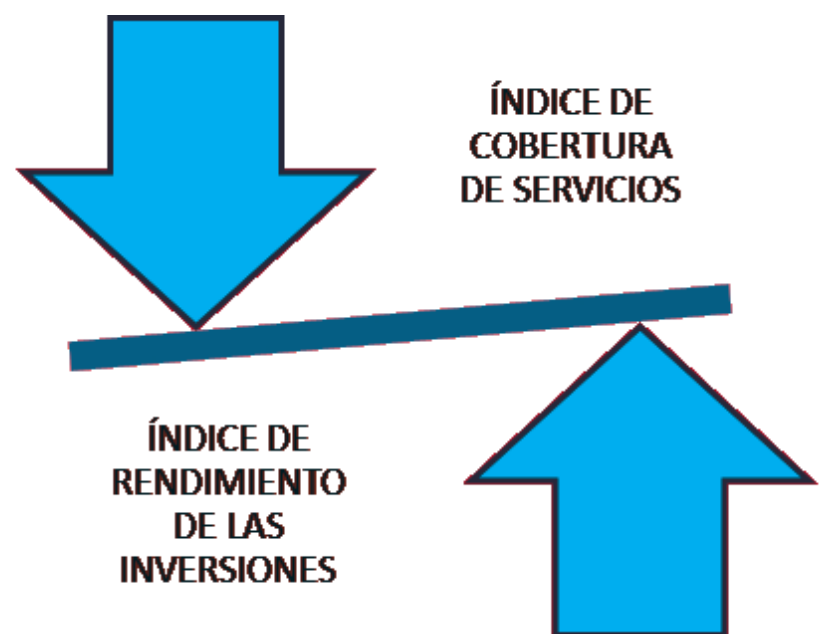

Fuente: elaboración propia.

Las metodologías propuestas, posibilitarán el estudio de los servicios públicos municipales desde 3 perspectivas: la interna, a través de los elementos y características básicas inherentes a cada uno de los servicios prestados; la económica, en relación a la inversión y costes en la prestación de los propios servicios; y una tercera desde el grado de cobertura de cada uno de los servicios analizados. Se consigue de esta forma, construir un instrumento idóneo para adaptar la gestión de las entidades locales a las necesidades de demanda de servicios, así como el establecimiento de una clasificación de los elementos prioritarios de cara a la inversión en base al grado de satisfacción que éstos producen en los ciudadanos.

\section{CONCLUSIONES}

La metodología diseñada, testada y presentada en líneas anteriores, cuya máxima expresión es el Índice de Cobertura de Servicios, presenta importantes potencialidades no sólo para los ciudadanos, cuya participación se convierte en elemento sobre el que debe pivotar la redefinición de los servicios, sino también para las instituciones y gestores que llevan a cabo la prestación de los mismos; puesto que:

- Fusiona elementos estructurales y perceptivos de cobertura. En la arquitectura de este índice agregado, tal y como se ha mencionado, intervienen una parte fija y común a todos los ayuntamientos de una misma franja poblacional, que es la que procede del análisis de la encuesta, o lo que es lo mismo, de la medición de la satisfacción ciudadana (elemento perceptivo); y una parte variable, procedente de los indicadores reales asociados a cada ayuntamiento y que definen el estado de cobertura de los servicios públicos prestados en cada uno de ellos (elemento estructural). Por todo ello, este índice no es un índice de satisfacción al uso.

- Determina niveles de servicio comparables entre municipios. El hecho de que la aplicación y estructura del ICS venga marcada por la clasificación poblacional de las unidades de análisis, los municipios, y que en su propia construcción matemática se tengan en cuenta tales diferencias; permite la comparabilidad de la cobertura facilitada entre ayuntamientos que comparten similitudes, dotando de realismo y validez a los resultados obtenidos.

- Permite repensar las políticas de inversión. Los resultados arrojados por el ICS pueden llegar a convertirse, en un mecanismo para el replanteamiento de las políticas de inversión que las entidades locales, bien los municipios o bien las diputaciones, llevan a cabo; identificando aquellos servicios sobre los cuáles, incrementos económicos no conllevan mejoras de los niveles de satisfacción y viceversa. De esta forma, se podrá obtener una óptima reasignación de recursos entre servicios y entre municipios.

- Facilita la transparencia y la rendición de cuentas. Como se expuso al principio de este trabajo, es vital para el conjunto de las administraciones públicas, y de forma especial en el caso de la administración local, el 
fomento del empleo de herramientas y/o instrumentos de medición que faciliten la evaluación de los servicios que facilitan a la ciudadanía, haciendo partícipe a esta última del proceso de gestión de los mismos.

- Se convierte en una herramienta de progreso, generadora de equilibrio. Finalmente, teniendo en cuenta la propia construcción del ICS, éste se revela como una herramienta de progreso, generadora de equilibrio; en el sentido en que favorece la mejora de la prestación de los servicios en aquellos ayuntamientos en los cuales es deficiente o inadecuada, manejando para ellos estándares de cobertura comparables, que tienden a minimizar la distorsión que pudieran ocasionar la presencia de valores extremos.

La herramienta que aquí se ha presentado, el ICS, se revela como una estructura de oportunidad para los políticos y gestores públicos que podrán emplearla como mecanismo para la evaluación de la prestación que de los servicios públicos se facilita a la ciudadanía, identificando los elementos críticos del servicio con el objetivo de minimizarlos; optimizando en la medida de lo posible, los recursos destinados a dicha prestación.

\section{BIBLIOGRAFÍA}

AEVAL (2014). Guía para la realización de estudios de análisis de la demanda y de evaluación de la satisfacción de los usuarios. Madrid: Ministerio de Hacienda y Administraciones Públicas. En línea: http://www.aeval.es/es/ difusion_y_comunicacion/publicaciones/Guias/Guias_Marco_General_Mejora_Calidad/guia_adyes_2014.html

Anderson, E.W.; Fornell, C. y Lehmann, D.R. (1994), “Customer satisfaction, market share, and profitability: findings from Sweden”. Journal of Marketing, 58: 53-66. DOI: $10.2307 / 1252310$

Andreassen, T.W. y Lervik, L. (1999), “Perceived relative attractiveness today and tomorrow as predictors of future repurchase intention”. Journal of Service Research, 2: 164-172. DOI: 10.1177/109467059922004.

Babakus, E. y Boller, G.W. (1992), “An empirical assessment of the SERVQUAL Scale”. Journal of Business Research, 24: 253-268. DOI: 10.1016/0148-2963(92)90022-4.

Batet i Lamaña, M. et al. (2001), Propuesta de indicadores básicos de gestión de servicios públicos locales. Barcelona: Fundació Carles Pi i Sunyer d’Estudis Autonómics i Locals. En línea: http://dialnet.unirioja.es/servlet/ articulo? codigo $=837795$.

Batet Lamaña, M. y Mora Puigví, A. (2001), «Indicadores de gestión de servicios públicos locales: una iniciativa desde Cataluña», en V. S. Local, Evaluación y control de políticas públicas. Gijón: Ayuntamiento de Gijón.

Bitner, M. (1990), «Evaluating service encounters: the effects of physical surroundings and emmployee responses», Journal of Marketing, 54: 69-82. DOI: 10.2307/1251871.

Bolton, E.N. y Drew, J.H. (1991), «A multistage model of customer's assessments of service quality and value», Journal of Consumer Research, 17: 375-384. En línea: http://www.jstor.org/stable/2626833.

Cadotte, E.; Woodruff, R. y Jenkins, R. (1987), «Expectations and norms in models of consumer satisfaction», Journal of Marketing Research, 24: 305-314. DOI: 10.2307/3151641.

Carman, J. (1990), «Consumer perceptions of service quality: an assessment of the SERVQUAL dimensions». Journal of Retailing, 66 (1): 33-55.

CFI. (2008), American Customer Satisfacion Index. Methodology report. Ann Arbor: Universtiy of Michigan.

Cronin, J.J. y Taylor, S.

- (1992), «Measuring service quality: a reexamination and extension», Journal of Marketing, 56 (3): 55-68. DOI: $10.2307 / 1252296$.

- (1994), «SERVPERF versus SERVQUAL: reconciling performance-based and percepcions-minus-expectations measurement of service quality», Journal of Marketing, 58 (1): 125-131. DOI: 10.2307/1252256.

Day, R. (1977), «Toward a process model of consumer satisfaction», en H. Hunt, Conceptualisation and measurement of consumer satisfaction and dissatisfaction. Cambridge: Marketing Science Institute.

Eklof, J. (2000), European customer satisfaction index pan-European telecommunication sector report based on the pilot studies 1999. Stockholm: European Organization of Quality and European Foundation for Quality Management. 
European Institute of Public Administration/European Public Administration Network (2008), European Primer on Customer Satisfaction Management. http://new.eupan.eu/files/repository/20101215131727_EU_Primer_ English_FINAL_LR.pdf

Evrard, Y. (1993), «La satisfaction de consommateurs: état de recherche», Reveu Française de Marketing, 144: $53-65$.

Fornell, C. (1992), «A National Customer Satisfaction Barometer: the Swedish experience», Journal of Marketing, 56: 6-21. DOI: $10.2307 / 1252129$.

Fornell, C. et al. (1996), «The American Customer Satisfaction Index: nature, purpose and findings», Journal of Marketing, 60: 7-18. DOI: 10.2307/1251898.

Giese, J.L. y Cote, J.A. (2000), «Defining consumer satisfaction», Academy of Marketing Science Review 1 (1): $1-22$.

Gil Saura, I. et al. (2004), «Calidad, Satisfacción y Valor del Servicio». Quadern de Treball (152): 3-53.

Gutiérrez Rodríguez, P. y García-Inés, M. (2008), El desarrollo de políticas públicas locales como garantes de la satisfacción de los ciudadanos. Barcelona: Fundación Alternativas.

Hunt, H. (1977), “Consumer satisfaction/dissatisfaction-overview and future research directions”, en H. Hunt, Conceptualisation and measurement of consumer satisfaction and dissatisfaction. Cambridge: Mass Marketing Science Institute.

Jaráiz Gulías, E.

- (2009), “La gestión de la atención primaria: confianza y satisfacción en el sistema sanitario de Galicia”, Revista de Investigaciones Políticas y Sociológicas, 8 (2): 69-84.

- (2011), La gestión sanitaria orientada al ciudadano: factores explicativos de la satisfacción ciudadana en la sanidad gallega. Rivera Otero, José M. (dir.), Universidad de Santiago de Compostela, Santiago de Compostela. http://hdl.handle.net/10347/3379.

Jaráiz Gulías, E., Lagares Diez, N. y Pereira López, M. (2013), “Los componentes de la satisfacción de los pacientes y su utilidad para la gestión hospitalaria”, Revista Española de Ciencia Política (32): 161-181.

López Hernández, A. y Ortiz Rodríguez, D. (2004), "Los indicadores de gestión y control de eficiencia del sector público”, Revista Española de Control Externo, 6 (18): 189-220.

López, A. M.; Navarro Galera, A. y Ortiz, D. (2001), Indicadores de gestión de servicios públicos locales: el proyecto SINIGAL, en V. S. Local, Evaluación y control de políticas públicas. Indicadores de gestión. Gijón: Ayuntamiento de Gijón.

Martínez-Tur, V., Peiró Silla, J.M. y Ramos, J. (2001), Calidad de servicio y satisfacción del cliente. Madrid: Editorial Síntesis.

Miller, J. (1977), “Exploring satisfaction modifying models, eliciting expectations, posing problems, and making meaningful measurements", en H. Hunt, Conceptualisation and measurement of consumer satisfaction and dissatisfaction. Cambridge: Mass Marketing Science Institute.

Nardo, M. et al.

- (2005), Tools for composite indicators building. Joint Research Centre-European Commission.

- (2005), Handbook on constructing composite indicators: methodology and user guide. Paris: OCDE.

Navarro Galera, A.; Ortiz Rodríguez, D. y López Hernández, A.M. (2006), “La aplicación de indicadores de gestión en la administración local: una propuesta para facilitar su viabilidad”, Logroño: IX Jornadas de Trabajo sobre Contabilidad Pública.

Oliver, R.

- (1980), "A cognitive model the antecedents and consequences of satisfaction decisions", Journal of Marketing Research, 17: 460-469. DOI: 10.2307/3150499. 
- (1981), "Measurement and evaluation of satisfaction processes in retail setting”, Journal of Retailing, 57 (3): $25-48$.

- (1993b), “A conceptual model of service quality and service satisfaction: compatible goals, different concepts”, en T. Swartz, y D. Bowen, Advances in services marketing and management: research and practice. Greenwich: JAI.

- (1993a), "Cognitive, affective and attribute bases of the satisfaction response", Journal of Consumer Research, 20: 418-430. DOI: 10.1086/209358.

- (1994), "Conceptual issues in the structural analisys of consumption, emotion, satisfaction, and quality: evidence in a service setting", Advances in Consumer Research: 16-22. http:/www.acrwebsite.org/search/ view-conference-proceedings.aspx? Id =7553.

- (1997), Satisfaction, a behavioral perspective on the consumer. Nueva York: McGraw-Hill.

Oliver, R. L. y Swan, J.E. (1989), "Consumer perceptions of interpersonal equity and satisfaction in transactions: a field sruvey approach”, Journal of Marketing, 53: 21-35. DOI: 10.2307/1251411.

Parasuraman, A.; Zeithmal, V. y Berry, L.

- (1988), "SERVQUAL: a multiple item scale for measuring consumer perceptions of service quality", Journal of Retailing, 64: 12-40.

- (1991), "Refinement and reassessment of the SERVQUAL scale”, Journal of Retailing, 67 (4): 420-450.

- (1994b), "Alternative scales for measuring service quality: a comparative assessment based on psychometric and diagnostic criteria", Journal of Retailing, 70 (3): 201-230. Doi: 10.1016/0022-4359(94)90033-7.

- (1994a), "Reassessment of expectations as a comparison standard in measuring service quality: implications for further research", Journal of Marketing, 58: 111-124. DOI: 10.2307/1252255.

Parasuraman, A.; Zeithmal, V. y Malhotra, A. (2005), “E-S-QUAL: A multiple-item scale for assessing electronic service quality”, Journal of Service Research, 7 (3): 213-233. DOI: 10.1177/1094670504271156.

Pereira López, M. (2013), Modelos de construcción de indicadores para la evaluación de los servicios públicos: definición y funcionamiento del Índice de Cobertura de Servicios (tesis doctoral), Rivera Otero, José M. (dir.), Universidad de Santiago de Compostela, Santiago de Compostela. http://hdl.handle.net/10347/9575.

Richard, M.D. y Allaway, A.W. (1993), "Service quality attributes and choice behavior", Journal of Services Marketing, 7: 69-68. DOI: 10.1108/08876049310026105.

Rust, R. T. y Oliver, R.L. (1994), "Service quality: insight and managerial implications from the frontier", en R. O. Rust, Service quality. New directions in theory and practice. Thousand Oaks, California: Sage Publications Inc.

Spreng, R.A. y MacKoy, R.D. (1996), “An empirical examination of a model of perceived service quality and satisfaction”, Journal of Retailing, 72: 201-214. DOI: 10.1016/S0022-4359(96)90014-7.

Teas, R. (1993), “Expectations, performance evaluation and consumer's perceptions of quality”, Journal of Marketing, 57: 18-34. DOI: $10.2307 / 1252216$.

Tse, D. y Wilton, P. (1988), “Models of consumer satisfaction formation: an extension”, Journal of Marketing Research, 25: 204-212. DOI: $10.2307 / 3172652$.

Van Ryzin, G.G. y DelPino, E. (2009), “Cómo escuchar, cómo aprender y cómo responder: las encuestas ciudadanas como una herramienta para la reinvención del gobierno”, Papeles de evaluación y calidad, 9: 1-15. http:// www.aeval.es/es/difusion_y_comunicacion/publicaciones/Papeles/Papeles_de_Evaluacixn_nx_9.html.

Westbrook, R. (1980), “A rating scale of measuring product/service satisfaction”, Journal of Marketing, 44: 68-85. DOI: $10.2307 / 1251232$.

Westbrook, R. A. y Reilly, D. (1983), "Value-precept disparity: an alternative to the disconfirmation of expectations theory of consumer satisfaction", en R. Bagozzi, Advances in Consumer Research. Ann Arbor: Association for Consumer Research. http://www.acrwebsite.org/search/view-conference-proceedings.aspx? Id=6120.

Zeithmal, V. y Bitner, M.J. (2002), Marketing de servicios. Un enfoque de integración del cliente a la empresa (Quinta ed.). México: McGraw-Hill. 